\title{
The Essence of Clinical Practice Guidelines for Ossification of Spinal Ligaments, 2019: 6. Diagnosis of OLF
}

\author{
Masao Koda \\ Department of Orthopaedic Surgery, University of Tsukuba, School of Medicine, Tsukuba, Japan
}

\author{
Keywords: \\ OLF, diagnosis, imaging

This article is the sixth part of the seven-article series, The Essence of Clinical Practice Guidelines for Ossification of Spinal Ligaments, published in the Spine Surgery and Related Research, Special Issue: Volume 5, Issue 5.

\section{Diagnosis of OLF}

\section{Summary}

- Primary symptoms include gait disturbance, lower limb motor and sensory disturbance, low back pain, and lower limb pain, none of which are specific to OLF.

- Neurological findings and signs vary depending on the level of nerve compression by OLF.

- While using plain X-ray is difficult to definitively diagnose OLF, plain X-ray can be useful for differential diagnosis, such as determining the presence or absence of DISH and OPLL.

- MRI and CT are useful for definite diagnosis and for examining the degree of spinal cord and cauda equina compression.

\section{Commentary}

While the symptoms of OLF differ depending on the level of nerve compression, the primary symptoms of spinal cord and cauda equina compression include gait disturbance, lower limb paralysis, sensory disturbance, low back pain, and lower limb pain ${ }^{1,2)}$, none of which are specific to OLF. Furthermore, some patients also present intermittent claudication as the chief complaint, and due care is needed to differentiate lumbar spinal stenosis and OLF.

At the thoracolumbar junction, which is a common site of
OLF, the epiconus is next to the conus medullaris and cauda equina. Thus, it is difficult to diagnose the level ${ }^{3)}$. The primary characteristics according to different levels are listed below:

- T10/11 intervertebral segment: Achilles tendon reflex (ATR) and patellar tendon reflex (PTR) are often accentuated (approximately 80\%) ${ }^{3,4)}$, and Beevor's sign can be positive $^{5)}$.

- T11/12 intervertebral segment: ATR can be accentuated, and PTR might be accentuated or attenuated. Low back pain with possible radiculopathy of the upper lumbar spine is characteristic ${ }^{6}$.

- T12/L1 intervertebral segment: Characteristic symptoms include muscle weakness (particularly of the tibialis anterior muscle), sensory disturbance of the lower extremities, and bladder and bowel disturbance ${ }^{3,4}$. The ATR is either less than normal or lost ${ }^{3,6}$.

- L1/2 intervertebral segment: Pain in the thigh and lateral knee is characteristic ${ }^{3)}$.

While OLF can be diagnosed by plain X-ray, it is often difficult to reach a definitive diagnosis using plain X-ray. However, plain X-ray is useful for the differential diagnosis of the presence or absence of DISH and OPLL. MRI is useful for the delineation of spinal cord compression and intramedullary signal intensity changes. OLF presents with hypointense signals on both T1-weighted and T2-weighted images; however, T2-weighted image is superior for identifying ossified lesions ${ }^{7,8)}$. Dural ossification can be detected by characteristic findings ("tram track sign" and "comma sign") of axial CT images ${ }^{9-12}$.

Conflicts of Interest: The author declares that there are

Corresponding author: Masao Koda, masaokod@gmail.com

Received: June 7, 2021, Accepted: July 8, 2021

Copyright (C) 2021 The Japanese Society for Spine Surgery and Related Research 
no relevant conflicts of interest.

This is a part of The Essence of Clinical Practice Guidelines for Ossification of Spinal Ligaments (2019), which is listed below.

1. Epidemiology of OPLL, written by Tomohiko Hasegawa, MD, PhD, Hamamatsu University, School of Medicine, Japan. https://doi.org/10.22603/ssrr.20210096

2. Pathology of OPLL, written by Takashi Kaito, MD, $\mathrm{PhD}$, Osaka University Graduate School of Medicine, Japan. https://doi.org/10.22603/ssrr.2021-0074

3. Diagnosis of OPLL, written by Hirotaka Chikuda, MD, $\mathrm{PhD}$, Gumma University, School of Medicine, Japan. https://doi.org/10.22603/ssrr.2021-0118

4. Treatment of Cervical OPLL, written by Toshitaka Yoshii, MD, PhD, Tokyo Medical and Dental University Hospital, Japan. https://doi.org/10.22603/ssrr.20210100

5. Treatment of Thoracic OPLL, written by Shiro Imagama, MD, PhD, Nagoya University Graduate School of Medicine, Japan. https://doi.org/10.22603/ssrr.20210095

6. Diagnosis of OLF, written by Masao Koda, MD, PhD, University of Tsukuba, Japan. https://doi.org/10.22603/ ssrr.2021-0116

7. Treatment of Thoracic OLF, written by Kanji Mori, MD, PhD, Shiga University of Medical Science, Japan. https://doi.org/10.22603/ssrr.2021-0094

The original version of this clinical practice guidelines appeared in Japanese as Sekichu Jintai Kokkashou Shinryo Guidelines 2019, published by the Japanese Orthopaedic Association and the Japanese Society for Spine Surgery and Related Research, and its translated version in English appeared in the Journal of Orthopaedic Science 26 (2021) 145.

\section{References}

1. He S, Hussain N, Li S, et al. Clinical and prognostic analysis of ossified ligamentum flavum in a Chinese population. J Neurosurg Spine. 2005;3(5):348-54.

2. Kang KC, Lee CS, Shin SK, et al. Ossification of the ligamentum flavum of the thoracic spine in the Korean population. J Neurosurg Spine. 2011;14(4):513-9.

3. Iwasaki M. The principles of spinal disorders. 2nd ed. Tokyo: Kanehara Shuppan; 2016. 52-9 p.

4. Fujimoto K, Kanchiku T, Imajo $Y$, et al. Neurologic findings caused by ossification of ligamentum flavum at the thoracolumbar junction. J Spinal Cord Med. 2017;40(3):316-20.

5. Beevor CE. The Croonian Lectures on muscular movements and their representation the central nervous system: delivered before the Royal College of Physicians of London. Br Med J. 1903;2 (2218):12-6.

6. Takenaka S, Kaito T, Hosono N, et al. Neurological manifestations of thoracic myelopathy. Arch Orthop Trauma Surg. 2014;134(7): 903-12.

7. Sugimura H, Kakitsubata Y, Suzuki Y, et al. MRI of ossification of ligamentum flavum. J Comput Assist Tomogr. 1992;16(1):73-6.

8. Yamashita Y, Takahashi M, Matsuno Y, et al. Spinal cord compression due to ossification of ligaments: MR imaging. Radiology. 1990;175(3):843-8.

9. Li B, Qiu G, Guo S, et al. Dural ossification associated with ossification of ligamentum flavum in the thoracic spine: a retrospective analysis. BMJ Open. 2016;6(12):e013887.

10. Sun XZ, Chen ZQ, Qi Q, et al. Diagnosis and treatment of ossification of the ligamentum flavum associated with dural ossification: clinical article. J Neurosurg Spine. 2011;15(4):386-92.

11. Zhou SY, Yuan B, Chen XS, et al. Imaging grading system for the diagnosis of dural ossification based on 102 segments of TOLF CT bone-window data. Sci Rep. 2017;7(1):2983.

12. Muthukumar N. Dural ossification in ossification of the ligamentum flavum: a preliminary report. Spine. 2009;34(24):2654-61.

Spine Surgery and Related Research is an Open Access journal distributed under the Creative Commons Attribution-NonCommercial-NoDerivatives 4.0 International License. To view the details of this license, please visit (https://creativeco mmons.org/licenses/by-nc-nd/4.0/). 